\title{
A Short, Non-Planetary, Microlensing Anomaly: Observations and Lightcurve Analysis of MACHO 99-BLG-47
}

\author{
M. D. Albrow ${ }^{1}$, J. An ${ }^{2}$, J.-P. Beaulieu ${ }^{3}$, J. A. R. Caldwell ${ }^{4}$, D. L. DePoy ${ }^{2}$, \\ M. Dominik ${ }^{5}$, B. S. Gaudi ${ }^{6,7}$, A. Gould ${ }^{2}$, J. Greenhill ${ }^{8}$, K. Hill ${ }^{8}$, S. Kane ${ }^{9}$, \\ R. Martin ${ }^{10}$, J. Menzies ${ }^{4}$, R. W. Pogge ${ }^{2}$, K. R. Pollard ${ }^{1}$, P. D. Sackett ${ }^{5}$, \\ K. C. Sahu ${ }^{11}$, P. Vermaak ${ }^{4}$, R. Watson ${ }^{8}$, and A. Williams ${ }^{10}$
}

(The PLANET Collaboration)

\begin{abstract}
We analyze PLANET and MACHO observations of MACHO 99-BLG-47, the first nearly-normal microlensing event for which high signal-to-noise-ratio data reveal a well-covered, short-duration anomaly. This anomaly occurs near the peak of the event. Short-duration anomalies near the peak of otherwise normal events are expected to arise both from extreme-separation (either very close or very wide), roughly equal-mass binary lenses, and from planetary systems. We show that the lens of MACHO 99-BLG-47 is in fact an extreme-separation binary, not a planetary system, thus demonstrating for the first time that these two important classes of events can be distinguished in practice. However, we find that the wide-binary and close-binary lens solutions fit the data equally well, and cannot be distinguished even at $\Delta \chi^{2}=1$. This degeneracy is qualitatively much more severe than the one identified for MACHO 98-SMC-1 because the present degeneracy spans two rather than one dimension in the magnification field and does not require significantly different blending fractions. In the appendix, we explore this result, and show that it is related to the symmetry in the lens equation.
\end{abstract}

Subject headings: binaries: general - gravitational lensing - planetary system

\section{Introduction}

The hallmark of planetary microlensing events is a short deviation from an otherwise normal, point-source/point-lens (hereafter PSPL) event. Mao \& Paczyński (1991) showed that extrasolar planets could be detected from such events, and Gould \& Loeb (1992) gave an explicit prescription for how the planet/star mass ratio $q(\ll 1)$ and the angular separation $d$ (in units of the angular Einstein radius $\theta_{\mathrm{E}}$ ) could be reconstructed by decomposing the event light curve into its "normal" and "perturbed" components.

Work during the ensuing decade has elucidated many additional subtleties of planetary light curves, but their fundamental characterization as briefly perturbed PSPL events has remained intact. Of particular importance in the present context, Griest \&

\footnotetext{
${ }^{1}$ Department of Physics \& Astronomy, University of Canterbury, Private Bag 4800, Christchurch, New Zealand

${ }^{2}$ Department of Astronomy, the Ohio State University, 140 West 18th Avenue, Columbus, OH 43210, USA

${ }^{3}$ Institut d'Astrophysique de Paris, INSU-CNRS, 98 bis Boulevard Arago, F 75014 Paris, France

${ }^{4}$ South African Astronomical Observatory, P.O. Box 9, Observatory, 7935 South Africa

${ }^{5}$ Kapteyn Institute, Rijksuniversiteit Groningen, Postbus 800, 9700 AV Groningen, The Netherlands

${ }^{6}$ School of Natural Sciences, Institute for Advanced Study, Einstein Drive, Princeton, NJ 08540, U.S.A.

${ }^{7}$ Hubble Fellow

${ }^{8}$ Physics Department, University of Tasmania, G.P.O. 252C, Hobart, Tasmania 7001, Australia

${ }^{9}$ School of Physics \& Astronomy, University of St. Andrews, North Haugh, St. Andrews, Fife KY16 9SS, UK

${ }^{10}$ Perth Observatory, Walnut Road, Bickley, Western Australia 6076, Australia

${ }^{11}$ Space Telescope Science Institute, 3700 San Martin Drive, Baltimore, MD 21218, USA
} 
Safizadeh (1998) showed that events with small impact parameter $\left(u_{0} \ll 1\right.$; where $u_{0}$ is the minimum separation between the source and the lens center of mass in units of $\theta_{\mathrm{E}}$ ) probe the so-called "central caustic" of the lens geometry, making them much more sensitive to the presence of planets than the larger impact-parameter events analyzed by Gould \& Loeb (1992), which probe the outer "planetary caustic".

These central-caustic events are of exceptional importance, even though they are intrinsically rare. They are rare simply because the central-caustic is much smaller than the planetary caustic, so the great majority of planet-induced deviations (of fixed fractional amplitude) are due to planetary caustics. However, the probability of detecting a planet is much greater in small impact-parameter events, partly because the source is guaranteed to pass close to the central caustic, which almost by definition is near the center of the lens geometry $(\boldsymbol{u}=0)$ and partly because even the sensitivity of planetary caustics is enhanced for $u_{0} \ll 1$. (Here $\boldsymbol{u}$ denotes the source position on the sky, normalized to $\theta_{\mathrm{E}}$, with respect to the lens center of mass.) By contrast, higher impact-parameter events miss the central caustic, and they are likely to miss the planetary caustic as well because it lies in a random position relative to the source trajectory. Because of their higher sensitivity to planets, and because they can be recognized in real time, low impact-parameter events are monitored more intensively than typical events by microlensing follow-up networks, which in turn further enhances their sensitivity to planets.

Central-caustic events, like their planetary-caustic cousins, involve a short deviation from an otherwise normal PSPL light curve. The major difference between these two classes of planetary events is that central-caustic anomalies always occur near the peak, whereas planetary-caustic perturbations can occur anywhere on the light curve, and are typically expected on the wings of the light curve. Of particular importance, for central-caustic events, there is no simple prescription for extracting $d$ and $q$ by decomposing the light curve into "normal" and "perturbed" components and it is unclear to what degree these two parameters are degenerate.

Another important, albeit accidental, discovery was that planets could give rise to perturbations that are not short compared to the event timescale. In the course of their search for planetary perturbations among 43 approximately PSPL events, Gaudi et al. (2002, see also Albrow et al. 2001b) found one event, OGLE-1999-BUL-36, that was asymmetric in a way that was consistent with the presence of a planet. They argued, however, that the asymmetry was also consistent with parallax effects induced by the Earth's motion around the Sun of the type analyzed by Gould, Miralda-Escudé, \& Bahcall (1994), and that in general it would be extremely difficult to distinguish between the two possible causes of such an asymmetry. They concluded that, in most cases, microlensing searches are not able to distinguish between parallax and a weak, asymmetric planetary perturbation, and consequently, all such "detections" should be ignored. This reduces the sensitivity of microlensing searches to planets, but only by an extremely small amount since, as Gaudi et al. (2002) showed, long-timescale asymmetric perturbations account for less than $\sim 1 \%$ of all planetary events. Hence, the long-timescale asymmetric events also confirm in a way the basic paradigm: planetary perturbations have short durations relative to the parent light curve, and in the rare cases for which they do not, they are not recognizable as a planetary perturbation anyway.

However, not all short timescale deviations are due to planets and therefore the mere detection of such an anomaly does not prove the presence of a planet. Gaudi \& Gould (1997) showed that close binaries $(d \ll 1)$ give rise to light curves that are virtually identical to PSPL events, except when the source comes very close to the lens center of mass $(|\boldsymbol{u}| \ll 1)$. Hence, for events with $u_{0} \ll 1$, the light curve looks "normal" except for a brief deviation near the peak. Qualitatively, this is exactly the same behavior as that of central-caustic planetary events. Similarly, light curves of wide-binary $(d \gg 1)$ events can also take the same form if one - and only one - of the caustics lies very close to the source's passage. Indeed, a close correspondence between a certain pair of close-binary and wide-binary events was discovered both theoretically (Dominik 1999) and observationally (Albrow et al. 1999; Afonso et al. 2000). It remains an open question under what conditions these various types of events can be distinguished from one another. If central-caustic planetary events could not be distinguished from close- and/or wide-binary events, it would seriously undermine planet searches in high-magnification events and hence would call into question the basic strategy adopted by microlensing follow-up groups (e.g., Albrow et al. 1998).

Here we analyze the light curve of the microlensing event MACHO 99-BLG-47, the first intensively monitored event with a short-lived, high signal-to-noise-ratio deviation from an otherwise normal PSPL light curve. We identify the lens as an extremeseparation binary rather than a planetary system, thereby showing that, at least in this case, the two classes can be clearly distinguished. We also show that both wide- and close-binary solutions fit the data equally well, implying that although planetary perturbations can be distinguished from those arising from extreme-separation binaries, the discrimination between very close and very wide binaries may be difficult in practice. 


\section{MACHO 99-BLG-47}

The initial alert for the microlensing event MACHO 99-BLG-47 was issued by the MACHO collaboration on 1999 July $23 .^{12}$ The event was located about $18^{\circ}$ from the Galactic center along the disk $\left(l=17^{\circ} 59^{\prime} 10^{\prime \prime}, b=-1^{\circ} 57^{\prime} 36^{\prime \prime}\right)$ and reported to be rather faint at baseline ( $V=21.5, R=20.3$ ). PLANET began monitoring MACHO 99-BLG-47 right after the electronic alert with the expectation that it would be a very high magnification event. PLANET detected anomalous behavior in the light curve during the first week of August, and subsequently issued an anomaly alert on 1999 August $4 .{ }^{13}$

\subsection{Observations and Data}

The PLANET light curve of MACHO 99-BLG-47 consists of observations made from three different southern sites: the Elizabeth $1 \mathrm{~m}$ at the South African Astronomical Observatory (SAAO), Sutherland, South Africa; the Canopus $1 \mathrm{~m}$ near Hobart, Tasmania, Australia; and the Yale/AURA/Lisbon/OSU (YALO) $1 \mathrm{~m}$ at Cerro Tololo Inter-American Observatory (CTIO), La Serena, Chile. The observations were carried out in two bands: $I$ (at all three sites) and $V$ (at SAAO only). The event was most intensely monitored in 1999 August during and following the photometric peak and the anomaly, but we obtained data through mid-September and also when the event was close to baseline early in the 2000 season as the source came out from behind the Sun. After the usual reductions, we perform photometry by two independent methods: a direct fit to the point spread function (PSF) using DoPHOT (Schechter, Mateo, \& Saha 1993) and difference imaging using ISIS (Alard \& Lupton 1998; Alard 2000). For details, see also Albrow et al. (2000a). Because of the different characteristics of the two methods and varying frame qualities, we recover a different number of photometric measurements. Before any elaborate efforts to clean and select the data, we have 303 points from DoPHOT photometry and 297 from ISIS photometry (Table 1) for the 1999 observations, as well as 8 points observed from YALO during early 2000 season (DoPHOT photometry only).

The photometric observations taken at SAAO are calibrated to the standard Johnson-Cousins system with respect to E-region stars (Menzies et al. 1989) observed contemporaneously with MACHO 99-BLG-47 at SAAO on 1999 July 31 . The instrumental magnitudes of observations from Canopus and YALO are not calibrated explicitly, but rather we allow independent source fluxes for different sites and bands in our modeling, which provide the relative photometric scalings among them.

We also include in our analysis the publicly available MACHO photometry, which comprises $77 V_{\mathrm{MACHO}}$ and $90 R_{\mathrm{MACHO}}$ points from the 1999 season as well as $213 V_{\mathrm{MACHO}}$ and $227 R_{\mathrm{MACHO}}$ points taken between 1995 and 1998 . The latter were used to constrain the baseline and to check for source variability. We find no evidence for such variability.

The difference photometry is placed on an absolute scale by a linear regression from the PSF-based flux (DoPHOT) to the differential flux (ISIS), after removing obvious faulty points from the two data sets based on the reports by each reduction/photometry package. We find that, except for the Canopus data, the residuals of the PSF-based flux from the regression line are strongly correlated with the size of the seeing disk, but that most of this correlation is removed by adding a linear seeing correction. For example, for the SAAO $I$-band observations, we detect the presence of a non-zero linear seeing dependence term of $\sim 1.7 F_{\text {base }} \operatorname{arcsec}^{-1}$ (or $\sim 10 F_{\mathrm{s}} \operatorname{arcsec}^{-1}$ ), with the signal to noise ratio being as large as $\sim 30$. Here $F_{\mathrm{s}}$ and $F_{\text {base }}$ are the net flux of the lensed source star alone and the total flux in the same PSF when there is no magnification.

In order to minimize the effects of known systematics, we optimize the data set by rejecting outliers and reevaluating the sizes of the photometric error bars. We also include a correction term for the correlation between the seeing and the blended flux that enters the same PSF with the lensed source when we fit the observed flux to a specific magnification model. These procedures are described in detail and fully justified in several earlier PLANET papers (Albrow et al. 2000a,b, 2001a,b; Gaudi et al. 2002). Briefly, we first construct an initial clean subset of the data by rejecting 3- $\sigma$ outliers with respect to the linear regression from DoPHOT to ISIS flux, and then determine a reference model based on this subset. Once we have a reference model, we include all the data and follow the same iterative procedure of outlier removal and error rescaling described in Albrow et al. (2001a). The final data set used for the subsequent analysis reported in the present paper contains 276 DoPHOT-reduced and 266 ISIS-reduced PLANET points as well as $166 \mathrm{MACHO}$ points (one $V_{\mathrm{MACHO}}$ point rejected), all from the 1999 season (Table 1). The data obtained during seasons other than 1999 (the 2000 season YALO $I$, and the $1995-1998$ season MACHO $V_{\mathrm{MACHO}}$ and

\footnotetext{
${ }^{12} \mathrm{ftp}: / /$ darkstar.astro.washington.edu/macho/Alert/99-BLG-47/

${ }^{13}$ http://www.astro.rug.nl//planet/MB99047.html
} 
$R_{\mathrm{MACHO}}$ bands) are included in the analysis as a combined single point for each set. In doing so, 4 data points (all $R_{\mathrm{MACHO}}$ in the 1998 season) are eliminated as outliers.

\section{Analysis and Model}

The light curve of MACHO 99-BLG-47 (Fig. 1) is that of a normal high-magnification PSPL event with a short-lived ( 3 days) deviation near the peak, of the type predicted for planets (Griest \& Safizadeh 1998) or binaries with extreme separations. We therefore develop a method for probing the space of lens geometries of this type and then search for the minimum $\chi^{2}$ within this space.

We begin by excising the anomalous points near the peak and fitting the remaining light curve to the degenerate form of the Paczyński (1986) profile that obtains in the high-magnification limit (Gould 1996),

$$
F(t)=\frac{F_{\text {peak }}}{\sqrt{1+\left(t-t_{0}\right)^{2} / t_{\text {eff }}^{2}}}+F_{\text {base }}
$$

Here $F(t)$ is the instantaneous flux, $F_{\text {peak }}$ is the peak flux above the baseline of the model, $F_{\text {base }}$ is the baseline flux, $t_{0}$ is the time of the peak, and $t_{\text {eff }}$ is the effective width of the light curve. These degenerate parameters are related to the standard parameters by $F_{\text {peak }}=F_{\mathrm{s}} / u_{0}, F_{\text {base }}=F_{\mathrm{s}}+F_{\mathrm{b}}$, and $t_{\text {eff }}=u_{0} t_{\mathrm{E}}$, where $t_{\mathrm{E}}$ is the Einstein timescale, i.e., the time required for the source to move an Einstein radius. We find $t_{0} \sim 2451393.6$ (Heliocentric Julian Date), $t_{\mathrm{eff}} \sim 1$ day, and that $F_{\text {peak }}$ roughly corresponds to $I_{\text {peak }} \sim 15.3$ (Fig. [1]). However, the exact values of those parameters are quite dependent on which points we excise. Note that though the baseline magnitude is measured to be $I_{\text {base }}=19.1$, there is at this point essentially no information of how the baseline flux divides into source flux $F_{\mathrm{s}}$ and blended flux $F_{\mathrm{b}}$, which is why it is necessary to fit the light curve to the degenerate profile (eq. [1]).

Next, we reinsert the excised points near the peak and note that the maximum (over-magnified) deviation from the degenerate fit is $\sim 0.5$ mag (Fig. 1). We then establish a set of initial trial event geometries as follows. For each diamond-shaped caustic produced by various geometries of $(d, q)$ pairs, we examine the magnification as a function of distance from the "caustic center" along each of the three directions defined by the four cusps of the caustic (one direction is redundant due to the reflection symmetry with respect to the binary axis). Here for computational simplicity, we use an analytic proxy point for the "caustic center," defined as follows. For close binaries, we adopt the center of mass of the lens system for the caustic center. On the other hand, for wide binaries, the position of one component of the binary shifted toward the other component by $\left[d\left(1+q^{-1}\right)\right]^{-1}$ is chosen as the caustic center. [These choices can be understood from the approximation developed in Appendix A. See also Dominik (1999).] Then, at each point $\boldsymbol{u}_{\bullet}$, the point of the cusp-axis crossing, we take the ratio of the actual magnification to that predicted for the corresponding PSPL lens: a point mass lens located at the caustic center with the same mass as either the whole binary (close binaries) or the nearest lens alone (wide binaries). We proceed with the search only if this ratio lies in the interval $[1.2,1.8]$. We then choose a source trajectory perpendicular to the cusp axis as an initial trial model, with $u_{0}=\left|\boldsymbol{u}_{\bullet}\right|$ and $t_{\mathrm{E}}=t_{\mathrm{eff}} /\left|\boldsymbol{u}_{\bullet}\right|$, and use four-parameter $\left(\alpha, t_{\mathrm{E}}, t_{0}\right.$, and $\left.u_{0}\right)$ downhill simplex (Press et al. 1992) to search for a best-fit model to minimize $\chi^{2}$, which is determined by a linear-flux fit to the model of lens magnifications (and thus the corresponding $F_{\mathrm{s}}$ and $F_{\mathrm{b}}$ are found immediately), subject to the constraint of fixed $(d, q)$. Figure 2 shows the result of this search (based on ISIS solutions) in a contour plot of $\chi^{2}$-surface over the $(d, q)$-space. The $\chi^{2}$ is rising around all the boundaries shown in Figure 2, except toward lower $q$. While the $\chi^{2}$-surface flattens with $\Delta \chi^{2} \simeq 32$ as the mass ratio $q$ becomes very small $(q \lesssim 0.01)$ for $d \sim 0.065$ and $d \sim 15$, we find no evidence of a decrease of the $\chi^{2}$ as $q$ becomes smaller than 0.01 . Rather it asymptotically approaches $\Delta \chi^{2} \simeq 32$.

We find well-localized minima of $\chi^{2}$ over the searched $(d, q)$-space, one of close binaries $(d \simeq 0.13)$ and the other of wide binaries $(d \simeq 11.3$ ), whose exact parameters depend slightly on whether we use the ISIS or DoPHOT photometry (Tables 2 and 3). We adopt the ISIS solutions in the subsequent discussion (and they are also what is shown in Fig. 2) because the light curve shows less scatter and consequently, the errors for the model parameter determinations are smaller. Despite the combination of the dense coverage near the peak by the PLANET data and the extensive baseline coverage by the MACHO data, the final two ISIS models (i.e., the close binary and the wide binary) are essentially indistinguishable: $\Delta \chi^{2}=0.6$ for 412 degrees of freedom, with the close binary having the lower $\chi^{2}$ of the two. In Appendix A, we discuss this degeneracy in further detail.

Finally, we also note that $u_{0} t_{\mathrm{E}}$ and $F_{\mathrm{s}} / u_{0}$ from the best-fit binary-lens model parameters are not quite the same as the $t_{\text {eff }}$ and $F_{\text {peak }}$ derived from the initial degenerate form of the light curve fit. These discrepancies are traceable to small differences between 
a true Paczyński (1986) curve and its degenerate approximation (eq. [1]), and are not due to differences between the binary and corresponding PSPL event, which are very small except around the peak. Since the parameters derived from equation (1) function only as seeds for simplex, and since the final $\chi^{2}$-surface is very well-behaved, these discrepancies in input values do not influence the final result.

\section{Discussion}

The mass ratios of the best fit models are $q=0.340 \pm 0.041$ (close binary) and $q=0.751 \pm 0.193$ (wide binary), which are well away from the regime of planetary companions $(q \lesssim 0.01)$. By comparison, the ratio of the duration of the anomalous portion of the light curve $\left(t_{\mathrm{anom}} \sim 3\right.$ days) to the Einstein timescale $\left(t_{\mathrm{E}} \sim 160 \text { days }\right)^{14}$ is $r=t_{\mathrm{anom}} / t_{\mathrm{E}} \sim 0.02$. For light curves perturbed by planetary caustics, one typically finds $q \sim r^{2}$ (Gould \& Loeb 1992). This relation clearly does not apply to the caustics of extreme-separation binaries.

Models with $q \leq 0.01$ (and $q \geq 100$ ) are formally rejected at $\Delta \chi^{2} \simeq 32$, which is significant but not in itself an overwhelming rejection of the planetary hypothesis. Hence, we examine the least $\chi^{2}$ model for $q=0.01\left(\Delta \chi^{2}=32.3\right)$ for its plausibility and the origin of statistical discriminating power. We discover that the difference of $\chi^{2}$ is mostly from the MACHO data between HJD 2451310 and 2451360, approximately 2 months prior to the photometric peak. However, we also find that the "planetary" model exhibits an extremely high peak magnification $\left(A_{\max } \sim 15000\right)$, and consequently, requires the event to be much longer $\left(t_{\mathrm{E}} \simeq 40 \mathrm{yr}\right)$ and more extremely blended $\left(I_{\mathrm{s}} \simeq 25.7, F_{\mathrm{s}} / F_{\text {base }} \simeq 0.2 \%\right)$ than the already unusually long and highly blended best-fit models. In addition, there is a clear trend of increasing peak magnification (and thus timescale and blend as well) as $q$ is lowered beyond 0.01 . This follows from the fact that the observed timescale of the anomaly in the light curve essentially fixes the source movement relative to the caustic. However, as $q$ becomes smaller, the size of the caustic relative to the Einstein ring shrinks, and therefore, the timescale of the event, that is the time required for the source to cross the Einstein ring, increases. (Note that this behavior causes a mild continuous degeneracy between $q$ and the blending.) These parameters determined for the "planetary" model are extremely contrived and highly improbable a priori. Furthermore, the timescale associated with the "planet" component, $t_{\mathrm{p}}=q^{1 / 2} t_{\mathrm{E}} \sim 4 \mathrm{yr}$ is much longer than that of typical stellar lenses, which further reduces the plausibility of the planetary interpretation. In summary, while in simple statistical terms, the star/planet scenario is not overwhelmingly disfavored relative to extreme-separation binaries, we nevertheless can conclude that it is highly unlikely that this event is due to a star/planet system.

From the standpoint of refining microlensing planet detection strategies, it is important to ask how one could have discriminated between the planetary and extreme-separation binary solutions with greater statistical significance. As noted above, most of the discriminating power came from the MACHO data points on the rising wing of the light curve, even though (or in a sense, because) these had the largest errors and the lowest density of the non-baseline coverage. That is, the precision PLANET photometry over the peak and falling wing "predicts" the rising wing for each of the models, but the noisier MACHO data can only roughly discriminate between these predictions. Hence, the key would have been to get better data on the rising part of the light curve. In practice this is difficult: the MACHO data are noisier exactly because they are survey data, and one does not know to monitor an event intensively until the light curve has actually started to rise.

The long duration of the event, $t_{\mathrm{E}} \simeq 160$ days (close binary) or $t_{\mathrm{E}} \simeq 220$ days (wide binary), may lead one to expect that the event would show some sign of parallax effects (Gould 1992; Mao 1999; Soszyński, et al. 2001; Bennett et al. 2001). Similarly, the close passage of the source to a cusp could in principle give rise to finite source effects (Gould 1994; Nemiroff \& Wickramasinghe 1994; Witt \& Mao 1994), as for example was recently observed in the case of EROS BLG-2000-5 (An et al. 2002). Indeed, the combination of parallax and finite-source effects permitted An et al. (2002) to measure the mass of a microlens for the first time. We have therefore searched for both parallax and finite source effects, but find no significant detection of either.

Finally, we examine the position of the source on the color-magnitude diagram (CMD; Fig. 3). The most prominent feature found in the CMD is a track of stars running diagonally in the same direction as would the main sequence. Since the field is in the Galactic disk, this feature is probably not a true main sequence, but rather likely to be a "reddening sequence", that is, an ensemble of mostly disk turnoff stars at progressively greater distances and correspondingly greater reddenings. The baseline

\footnotetext{
${ }^{14}$ While the value here is for the close binary, the value for the wide binary is essentially the same because the relevant mass of the corresponding point-mass lens for the wide binary is not the combined mass but the mass of the single component that the source passes by.
} 
"star" (combined light of the source and blended light) lies within this sequence toward its faint/red end although its position is seeing-dependent because of the seeing-correction term. The position indicated in the CMD is plotted assuming median seeing. However, the source itself $\left[I_{\mathrm{s}}=20.9,(V-I)_{\mathrm{s}}=1.94\right]$ lies $\sim 2$ mag below the baseline "star," in a region of the CMD that is well below the threshold of detection. If the baseline were mainly composed of source light, and the "blended light" were simply source light that had been falsely attributed to blending by a wrong model, then the real source would lie within the well populated "reddening sequence", and the timescale would be much shorter, $t_{\mathrm{E}} \sim 30$ days. We therefore searched for solutions with little or no blending. However, we find that these are ruled out with $\Delta \chi^{2}=2440$. Furthermore, the best-fit models with no blended light are still extreme-separation binaries and not planetary systems. Since we have only a crude understanding of the $\mathrm{CMD}$, no strong argument can be made that the position of the source is implausible. If, in fact, the observed track of stars in Figure 3 is really the reddening sequence of disk turnoff stars, the source position is consistent with a low-mass main-sequence star lying $\gtrsim 2$ mag below the turnoff. This would also explain the lack of finite source effect. Moreover, we note that in disk fields, long events are not at all uncommon (Derue et al. 2001) because the observer, source, and lens are all moving with roughly the same velocity.

Regarding the large amount of blended light required to fit the observed light curve, we note that the highly significant seeing effect in the PSF-based photometry (see $\S 2.1$ ) is also evidence that the event is strongly blended. In addition to the direct confirmation of the strong seeing effect from the comparison between the differential flux and the PSF-based flux measurements, we independently detect a significant seeing correction when we fit the observed flux to the magnification model. For SAAO $I$-band observation, the seeing correction determined from the model fit using DoPHOT flux is slightly smaller $\left(\sim 9 F_{\mathrm{S}} \operatorname{arcsec}^{-1}\right)$ than the value derived from the regression between DoPHOT and ISIS flux. The seeing correction derived by fitting ISIS data to the model is basically consistent with zero.

\section{Binary Lens vs. Binary Source}

Multiple-peak events like the one seen in Figure 1 can be caused by a binary source (Griest \& Hu 1992) rather than a binary lens. In general, one expects that such events will be chromatic, since the colors of the two sources will not usually be the same. By contrast, MACHO 99-BLG-47 is achromatic: the difference in color of the two wings of the light curve is $\Delta V-I=0.01 \pm 0.05$. In the limit that one component of the binary is completely dark, the event will be achromatic, and yet can have still have multiple peaks caused by rotation of the binary during the event. In this case, however, there will be a series of roughly equally spaced peaks that gradually die out as the event declines (Han \& Gould 1997), contrary to the distinct double-peaked behavior seen in Figure 1. Hence, a binary-source explanation appears a priori implausible.

Nevertheless, it is possible in principle that the source has two components of nearly identical color. We search for binarysource models, with either static or slowly moving components, but find that the best fit model has $\Delta \chi^{2}=1167$, which clearly rules out such models. The basic problem is that the observed second peak is very sharp given its height and the timescale of the declining light curve. Binary-source light curves that fit these latter features tend to have a width that is closer to that of the dotted curve in Figure 1. Recall that this curve represents a single-lens degenerate fit to the non-peak data. We conclude that the explanation for the double-peaked behavior of the light curve is that it is a binary-lens rather than a binary-source event.

\section{Conclusion}

MACHO 99-BLG-47 is the first microlensing event with a short-lived, high signal-to-noise-ratio anomaly, characteristics that could betray the existence of a planet around the lensing star. Nevertheless, we conclude that the lens of MACHO 99-BLG-47 is not a planetary system, but an extreme-separation (very close or very wide) binary composed of components of similar mass, based on the result of the light curve fit as well as the extreme value of event duration and blending fraction required for any plausible "planetary" fit.

\section{ACKNOWLEDGEMENTS}

We thank the MACHO collaboration for providing the initial alert for this event and for allowing us to use their photometric data for the modeling. We thank Joachim Wambsganss for his comments on and a thorough reading of the draft version of this paper. 
We are especially grateful to the observatories that support our science (Canopus, CTIO, SAAO) via the generous allocations of time that make this work possible. The operation of Canopus Observatory is in part supported by the financial contribution from Mr. David Warren. PLANET acknowledges financial support via award GBE 614-21-009 from de Nederlandse Organisatie voor Wetenschappelijk Onderzoek (NWO), the Marie Curie Fellowship ERBFMBICT972457 from the European Union (EU), "coup de pouce 1999" award from le Ministère de l'Éducation nationale, de la Recherche et de la Technologie, Département Terre-Univers-Environnement, the Presidential Fellowship from the Graduate School of the Ohio State University, a Hubble Fellowship from the Space Telescope Science Institute (STScI), which is operated by the Association of Universities for Research in Astronomy (AURA), Inc., under NASA contract NAS5-26555, the Jet Propulsion Laboratory (JPL) contract 1226901, grants AST 97-27520 and AST 95-30619 from the National Science Foundation (NSF), and grants NAG5-7589 and NAG5-10678 from the National Aeronautics and Space Administration (NASA). This paper utilizes public domain data obtained by the MACHO Project, jointly funded by the US Department of Energy through the University of California, Lawrence Livermore National Laboratory under contract No. W-7405-Eng-48, by the NSF through the Center for Particle Astrophysics of the University of California under cooperative agreement AST-8809616, and by the Mount Stromlo and Siding Spring Observatory, part of the Australian National University.

\section{A. Close and Wide Binary Correspondence}

Dominik (1999) noticed that the Chang \& Refsdal $(1979,1984)$ lens (CRL; see also Schneider, Ehlers, \& Falco 1992) well approximates the binary lens system in the vicinity of each individual lens component of extreme wide-separation $(d \gg 1)$ binaries and in the vicinity of the secondary of extreme close-separation $(d \ll 1)$ binaries while the quadrupole lens (QL) approximation works nicely near the center of mass of extreme close-separation binaries. He further showed that the size and shape of the caustics and the behavior of the magnification field associated with them are very similar among the true binary lenses, the CRL approximation, and the QL approximation if the shear, $\gamma$, of the CRL and the absolute eigenvalue of the quadrupole moment tensor, $\hat{Q}$ (for simplicity, hereafter $\hat{Q}$ will be just referred to "the quadrupole moment"), of the QL are both very small and their numerical values are close to each other $(\gamma \simeq \hat{Q} \ll 1)$. These findings also imply the existence of a correspondence between a wide binary with $\gamma=d_{w}^{-2} q_{w}\left(1+q_{w}\right)^{-1}$ and a close binary with $\hat{Q}=d_{c}^{2} q_{c}\left(1+q_{c}\right)^{-2}$, sometimes referred to as a $d \leftrightarrow d^{-1}$ correspondence. (Here and throughout this appendix, we use the subscript ${ }_{c}$ and ${ }_{w}$ to distinguish the parameters associated with the close and wide binaries.) Here we rederive the basic result for this correspondence, and explore it more thoroughly.

The general form of the binary lens mapping equation can be expressed in notation utilizing complex numbers (Witt 1990),

$$
\zeta=z-\frac{\epsilon_{1}}{\bar{z}-\bar{z}_{1}}-\frac{\epsilon_{2}}{\bar{z}-\bar{z}_{2}},
$$

and the magnification associated with a single image can be found by,

$$
A=\left(1-\left|\frac{\partial \zeta}{\partial \bar{z}}\right|^{2}\right)^{-1}
$$

where $\zeta, z, z_{1}$, and $z_{2}$ are the positions of the source, the image, and the two lens components normalized by the Einstein radius of some mass, and $\epsilon_{1}$ and $\epsilon_{2}$ are the masses of lens components in units of the same mass. For the extreme close-binary case, if one chooses the center of mass as the origin and the binary axis as the real axis and sets the combined mass to be unity, then $z_{1}=d_{c} \epsilon_{2}, z_{2}=-d_{c} \epsilon_{1}, \epsilon_{1}=\left(1+q_{c}\right)^{-1}$, and $\epsilon_{2}=q_{c}\left(1+q_{c}\right)^{-1}$, and the lens equation (A1) becomes

$$
\begin{aligned}
\zeta & =z-\frac{\epsilon_{1}}{\bar{z}-d_{c} \epsilon_{2}}-\frac{\epsilon_{2}}{\bar{z}+d_{c} \epsilon_{1}} \\
& \approx z-\frac{1}{\bar{z}}-\frac{d_{c}^{2} \epsilon_{1} \epsilon_{2}}{\bar{z}^{3}}+\frac{d_{c}^{3} \epsilon_{1} \epsilon_{2}\left(\epsilon_{1}-\epsilon_{2}\right)}{\bar{z}^{4}}+\cdots \quad\left(d_{c} \ll|z|\right) .
\end{aligned}
$$

Here the monopole term $\left(\bar{z}^{-1}\right)$ is the same as for a PSPL and the first non-PSPL term is the quadrupole $\left(\bar{z}^{-3}\right)$, which acts as the main perturbation to the PSPL if the quadrupole moment is small; $\hat{Q}=d_{c}^{2} \epsilon_{1} \epsilon_{2}=d_{c}^{2} q_{c}\left(1+q_{c}\right)^{-2} \ll 1$. For a given source position $\zeta$, let the image position for the PSPL be $z_{0}$. Then, under the perturbative approach, the image for the QL approximation is found at $z=z_{0}+\delta z_{c}$, where $\left|\delta z_{c}\right| \ll\left|z_{0}\right| \sim 1$. Using the fact that $z_{0}$ is the image position corresponding to $\zeta$ for the PSPL (i.e., $\zeta=z_{0}-\bar{z}_{0}^{-1}$ ), one obtains

$$
\delta z_{c} \approx \hat{Q}\left(1-\frac{1}{\left|z_{0}\right|^{4}}\right)^{-1}\left[\left(\frac{1}{\bar{z}_{0}^{3}}-\frac{1}{z_{0}^{3} \bar{z}_{0}^{2}}\right)+\left(\frac{1}{z_{0}^{4} \bar{z}_{0}^{2}}-\frac{1}{\bar{z}_{0}^{4}}\right) \frac{1-q_{c}}{1+q_{c}} d_{c}+\cdots\right],
$$


from equation (A4) and taking only terms linear in $\delta z_{c}$ and $\hat{Q}$. Here, we note that $\hat{Q} \sim \mathcal{O}\left(d_{c}^{2}\right)$ so that the second term in the bracket is lower order than $\hat{Q}^{2}$. In order to find the magnification for this image, we differentiate equation (A4), and find,

$$
\frac{\partial \zeta}{\partial \bar{z}} \approx \frac{1}{\bar{z}^{2}}+\frac{3 \hat{Q}}{\bar{z}^{4}}\left[1-\frac{4\left(1-q_{c}\right)}{3\left(1+q_{c}\right)} \frac{d_{c}}{\bar{z}}+\cdots\right]
$$

Then substituting $z=z_{0}+\delta z_{c}$, for which $\delta z_{c}$ is given by equation (A5), into equation (A6), we obtain,

$$
\left|\frac{\partial \zeta}{\partial \bar{z}}\right|^{2} \approx \frac{1}{\left|z_{0}\right|^{4}}+\hat{Q}\left[\frac{3\left|z_{0}\right|^{4}-2\left|z_{0}\right|^{2}-1}{\left|z_{0}\right|^{8}-\left|z_{0}\right|^{4}}\left(\frac{1}{z_{0}^{2}}+\frac{1}{\bar{z}_{0}^{2}}\right)-\frac{4\left|z_{0}\right|^{4}-2\left|z_{0}\right|^{2}-2}{\left|z_{0}\right|^{8}-\left|z_{0}\right|^{4}}\left(\frac{1}{z_{0}^{3}}+\frac{1}{\bar{z}_{0}^{3}}\right) \frac{1-q_{c}}{1+q_{c}} d_{c}+\cdots\right] .
$$

Note that the total magnification for the given source position is usually dominated by one or two images found close to the critical curve. Thus, we consider only the case for which the non-perturbed PSPL images lie close to the unit circle, so we have $\left|z_{0}\right|=1+\Delta$ and $\Delta \ll 1$. Then, we find the expression for the inverse magnification for the QL approximation (up to the order of $\left.d_{c}^{3}\right)$

$$
\begin{aligned}
A^{-1} & \approx\left|4 \Delta-2 \hat{Q}\left(\frac{1}{z_{0}^{2}}+\frac{1}{\bar{z}_{0}^{2}}\right)+3 \hat{Q}\left(\frac{1}{z_{0}^{3}}+\frac{1}{\bar{z}_{0}^{3}}\right) \frac{1-q_{c}}{1+q_{c}} d_{c}\right| \\
& =4\left|\left(\left|z_{0}\right|-1\right)-\hat{Q} \Re\left(z_{0}^{-2}\right)+\frac{3\left(1-q_{c}\right)}{2\left(1+q_{c}\right)} d_{c} \hat{Q} \Re\left(z_{0}^{-3}\right)\right|
\end{aligned}
$$

For the extreme wide-binary case, one can rewrite the lens equation (A1) as

$$
\begin{aligned}
\zeta & =z-\frac{1}{\bar{z}}-\frac{q_{w}}{\bar{z}+d_{1}} \\
& \approx z-\frac{1}{\bar{z}}-\frac{q_{w}}{d_{1}}+\frac{q_{w}}{d_{1}^{2}} \bar{z}-\frac{q_{w}}{d_{1}^{3}} \bar{z}^{2}+\cdots \quad\left(d_{w} \gg|z|\right) .
\end{aligned}
$$

Here the position and the mass of the first lens component are the origin and the unit mass so that $z_{1}=0, z_{2}=-d_{1}, \epsilon_{1}=1$, $\epsilon_{2}=q_{w}$, and $d_{1}=\left(1+q_{w}\right)^{1 / 2} d_{w}$. We note that, apart from the constant translation, the first non-PSPL term here is essentially the shear, $\gamma=q_{w} d_{1}^{-2}=q_{w} d_{w}^{-2}\left(1+q_{w}\right)^{-1}$ for the CRL approximation. Analogous to the extreme close binary, if $\gamma \ll 1$, the image position for the CRL approximation can be found by the perturbative approach, but here the corresponding PSPL source position would be $\zeta+q_{w} d_{w}^{-1}=z_{0}-\bar{z}_{0}^{-1}$. Then, the image deviation $\delta z_{w}=z-z_{0}$ of CRL from PSPL is

$$
\delta z_{w} \approx \gamma\left(1-\frac{1}{\left|z_{0}\right|^{4}}\right)^{-1}\left[\left(\frac{z_{0}}{\bar{z}_{0}^{2}}-\bar{z}_{0}\right)+\left(\bar{z}_{0}^{2}-\frac{z_{0}^{2}}{\bar{z}_{0}^{2}}\right) \frac{1}{\left(1+q_{w}\right)^{1 / 2} d_{w}}+\cdots\right]
$$

Using this result and the derivative of equation (A10),

$$
\frac{\partial \zeta}{\partial \bar{z}} \approx \frac{1}{\bar{z}^{2}}+\gamma\left[1-\frac{2}{\left(1+q_{w}\right)^{1 / 2}} \frac{\bar{z}}{d_{c}}+\cdots\right]
$$

we find

$$
\left|\frac{\partial \zeta}{\partial \bar{z}}\right|^{2} \approx \frac{1}{\left|z_{0}\right|^{4}}+\gamma\left[\frac{\left|z_{0}\right|^{4}+2\left|z_{0}\right|^{2}-3}{\left|z_{0}\right|^{4}-1}\left(\frac{1}{z_{0}^{2}}+\frac{1}{\bar{z}_{0}^{2}}\right)-\frac{2\left|z_{0}\right|^{6}+2\left|z_{0}\right|^{4}-4\left|z_{0}\right|^{2}}{\left|z_{0}\right|^{4}-1}\left(\frac{1}{z_{0}^{3}}+\frac{1}{\bar{z}_{0}^{3}}\right) \frac{1}{\left(1+q_{w}\right)^{1 / 2} d_{w}}+\cdot\right] .
$$

From the same argument used for the QL approximation of the extreme close binary, we can set $\left|z_{0}\right|=1+\Delta$, and then the inverse magnification for the CRL approximation (up to the order of $d_{w}^{-3}$ ) is

$$
\begin{aligned}
A^{-1} & \approx\left|4 \Delta-2 \gamma\left(\frac{1}{z_{0}^{2}}+\frac{1}{\bar{z}_{0}^{2}}\right)+3 \gamma\left(\frac{1}{z_{0}^{3}}+\frac{1}{\bar{z}_{0}^{3}}\right) \frac{1}{\left(1+q_{w}\right)^{1 / 2} d_{w}}\right| \\
& =4\left|\left(\left|z_{0}\right|-1\right)-\gamma \Re\left(z_{0}^{-2}\right)+\frac{3}{2\left(1+q_{w}\right)^{1 / 2}} \frac{\gamma}{d_{w}} \Re\left(z_{0}^{-3}\right)\right| .
\end{aligned}
$$

By comparing equations (A8) and (A14), we therefore establish the magnification correspondence (up to the order of $d_{c}^{2} \sim d_{w}^{-2}$ ) between the close binary with $\hat{Q}=d_{c}^{2} q_{c}\left(1+q_{c}\right)^{-2}$ and the wide binary with $\gamma=d_{w}^{-2} q_{w}\left(1+q_{w}\right)^{-1}$ when $\hat{Q} \simeq \gamma \ll 1$.

For the two PLANET ISIS models for MACHO 99-BLG-47, we obtain $\hat{Q}=3.40 \times 10^{-3}$ for the close binary solution $\left(d_{c}, q_{c}\right)=(0.134,0.340)$ and $\gamma=3.35 \times 10^{-3}$ for the wide binary solution $\left(d_{w}, q_{w}\right)=(11.31,0.751)$. Hence, the observed 
degeneracy between the two PLANET models (see $\S 3$ and Tables 2 and 3 ) is the clearest example of this type of correspondence observed so far. While Afonso et al. (2000) reported that two different binary lens models, one of a close binary and the other of a wide binary, can fit the observed light curve of the (caustic-crossing) binary lens event, MACHO 98-SMC-1, ${ }^{15}$ one can infer from their figure 8, that the degeneracy between the two models exists only for the specific light curves (which is essentially a particular one dimensional slice of the magnification field over the source plane) but not for the magnification field in the neighborhood of the caustic as a whole. This is obvious from the relative rotation of the two caustics. In addition, the source magnitudes for the two models of MACHO 98-SMC-1 differ by $\sim 0.18$ mag. By contrast, the difference of the predicted $I_{\mathrm{s}}$ between the two models of MACHO 99-BLG-47 is only $\sim 0.02$ mag. In fact, we get $\hat{Q}=6.5 \times 10^{-2}$ (the close binary) and $\gamma=1.8 \times 10^{-2}$ (the wide binary) for the two models of MACHO 98-SMC-1, and thus, although the degeneracy of the MACHO 98-SMC-1 light curve is somehow related to the correspondence of $d \leftrightarrow d^{-1}$, it cannot be completely explained simply by the argument in this appendix, and it should be investigated further for its origin. On the other hand, the degeneracy of MACHO 99-BLG-47 is the first definitive observational case of the correspondence between extreme separation binaries. This can be also seen in Figure 4, which illustrates the similarity between the magnification fields for the two models of MACHO 99-BLG-47.

The very low $\Delta \chi^{2}$ between the two solutions despite the excellent data implies that it is extraordinarily difficult to break this degeneracy with photometric data. Gould \& Han (2000) showed that for MACHO 98-SMC-1 the two solutions were also astrometrically degenerate, at least for data streams lying within a few $t_{\mathrm{E}}$ of the peak. They argued that this astrometric degeneracy, like the corresponding photometric degeneracy, was rooted in the lens equation. However, as shown in this appendix, the correspondence between the equations describing close- and wide-binaries is purely local (See also Dominik 2001). For example, there is a constant-offset term in equation (A10), which does not give rise to any local photometric or astrometric effects, but which must "disappear" at late times. Hence there must be a late-time astrometric shift between the two solutions. Such a shift was noted explicitly by Gould \& Han (2000) for the case of MACHO 98-SMC-1, and these are likely to be a generic feature of close/wide corresponding pairs of solutions.

We also plot the lines of $(d, q)$ pairs that have the same shear $\gamma$ as the best-fit wide-binary model or the same quadrupole moment $\hat{Q}$ as the best-fit close-binary model on $\chi^{2}$-surface contour plot shown in Figure 2. While the iso-shear line for wide binaries lies nearly parallel to the direction of the principal conjugate near the best-fit model, it is clear from the figure that the condition of $\hat{Q} \simeq \gamma \ll 1$ alone does not define the observed well-defined two-fold degeneracy, which involves the additional correspondence between higher order terms beyond the quadrupole moment $\left(\sim d_{c}^{2}\right)$ and the pure shear $\left(\sim d_{w}^{-2}\right)$. Further comparison between equations (A8) and (A14) indicates that there exists a magnification correspondence up to the order of $d_{c}^{3} \sim d_{w}^{-3}$ if the condition $d_{c}\left(1-q_{c}\right)\left(1+q_{c}\right)^{-1}=d_{w}^{-1}\left(1+q_{w}\right)^{-1 / 2}$ is also satisfied in addition to $\hat{Q}=\gamma \ll 1$. We find that, for the two PLANET models, $d_{c}\left(1-q_{c}\right)\left(1+q_{c}\right)^{-1}=6.6 \times 10^{-2}$ (the close binary) and $d_{w}^{-1}\left(1+q_{w}\right)^{-1 / 2}=6.7 \times 10^{-2}$ (the wide binary). Hence, we conclude that, in fact, these two conditions,

$$
\begin{gathered}
d_{c}^{2} d_{w}^{2}\left(1+q_{w}\right)=\frac{q_{w}}{q_{c}}\left(1+q_{c}\right)^{2}, \\
d_{c} d_{w}\left(1+q_{w}\right)^{1 / 2}=\frac{1+q_{c}}{1-q_{c}},
\end{gathered}
$$

define a unique correspondence between two extreme separation binaries. We also note that the images (not shown) of the iso$\Delta \chi^{2}$ contours for the close binary models of MACHO 99-BLG-47 under the mapping defined by the above two relations follows extremely closely the corresponding iso- $\Delta \chi^{2}$ contour for wide binary models, except for the difference of $\Delta \chi^{2} \simeq 0.6$ offset between two solutions.

\footnotetext{
${ }^{15}$ Albrow et al. (2001a) also found a similar degeneracy while they modeled the light curve of OGLE-1999-BUL-23, but they could discriminate the two models to better than $\Delta \chi^{2} \simeq 128$.
} 


\section{REFERENCES}

Afonso, C., et al. 2000, ApJ, 532, 340

Alard, C. 2000, A\&AS, 144, 363

Alard, C., \& Lupton, R. H. 1998, ApJ, 503, 325

Albrow, M. D., et al. 1998, ApJ, 509, 687

Albrow, M. D., et al. 1999, ApJ, 522, 1022

Albrow, M. D., et al. 2000a, ApJ, 534, 894

Albrow, M. D., et al. 2000b, ApJ, 535, 176

Albrow, M. D., et al. 2001a, ApJ, 549, 759

Albrow, M. D., et al. 2001b, ApJ, 556, L113

An, J. H., et al. 2002, ApJ, 572, 000

Bennett, D. P., et al. 2001, ApJ, submitted (astro-ph/0109467)

Chang, K., \& Refsdal, S. 1979, Nature, 282, 561

Chang, K., \& Refsdal, S. 1984, A\&A, 132, 168

Derue, F., et al. 2001, A\&A, 373, 126

Dominik, M. 1999, A\&A, 349, 108

Dominik, M. 2001, in ASP Conf. Ser. 237, Gravitational Lensing: Recent Progress and Future Goals, ed. T. G. Brainerd \& C. S. Kochanek (San Francisco, CA: Astronomical Society of the Pacific)

Gaudi, B. S., et al. 2002, ApJ, 566, 463

Gaudi, B. S., \& Gould, A. 1997, ApJ, 482, 83

Gould, A. 1992, ApJ, 392, 442

Gould, A. 1994, ApJ, 421, L71

Gould, A. 1996, ApJ, 470, 201

Gould, A., \& Han, C. 2000, ApJ, 538, 653

Gould, A., \& Loeb, A. 1992, ApJ, 396, 104

Gould, A., Miralda-Escudé, J., \& Bahcall, J. N. 1994, ApJ, 423, L105

Griest, K., \& Hu, W. 1992, ApJ, 397, 362

Griest, K., \& Safizadeh, N. 1998, ApJ, 500, 37

Han, C., \& Gould, A. 1997, ApJ, 480,196

Mao, S. 1999, A\&A, 350, L19

Mao, S., \& Paczyński, B. 1991, ApJ, 374, L37

Menzies, J. W., Cousins, A. W. J., Banfield, R. M., \& Laing, J. D. 1989, SAAO Circ., 13, 1

Nemiroff, R. J., \& Wickramasinghe, W. A. D. T. 1994, ApJ, 424, L21

Paczyński, B. 1986, ApJ, 304, 1 
Press, W. H., Teukolsky, S. A., Vetterling, W. T., \& Flannery, B. P. 1982, Numerical Recipes (Cambridge: Cambridge University Press)

Schneider, P., Ehlers, J., \& Falco, E. E. 1992, Gravitational Lenses (Berlin: Springer-Verlag)

Schechter, P. L., Mateo, M., \& Saha, A. 1993, PASP, 105, 1342

Soszyński, I., et al. 2001, ApJ, 552, 731

Witt, H. J. 1990, A\&A, 236, 311

Witt, H. J., \& Mao, S. 1994, ApJ, 430, 505 
Table 1. Photometric data of MACHO 99-BLG-47

\begin{tabular}{|c|c|c|c|c|c|}
\hline telescope & filter & $\#$ of total points & \# of points analyzed & $\begin{array}{l}\text { median seeing } \\
\quad(\operatorname{arcsec})\end{array}$ & error rescaling factor \\
\hline \multicolumn{6}{|c|}{ PLANET (DoPHOT) } \\
\hline Elizabeth $1 \mathrm{~m}$ & $I$ & 98 & 88 & 1.81 & 1.19 \\
\hline$\ldots$ & $V$ & 26 & 24 & 1.79 & 1.02 \\
\hline Canopus $1 \mathrm{~m}$ & $I$ & 51 & 42 & 2.71 & 1.43 \\
\hline YALO $1 \mathrm{~m}$ & $I$ & 128 & 122 & 1.96 & 0.772 \\
\hline \multicolumn{6}{|c|}{ PLANET (ISIS) } \\
\hline Elizabeth $1 \mathrm{~m}$ & $I$ & 105 & 98 & 1.65 & 1.35 \\
\hline$\cdots$ & $V$ & 21 & 20 & 1.96 & 1.83 \\
\hline Canopus $1 \mathrm{~m}$ & $I$ & 57 & 38 & 2.59 & 1.75 \\
\hline YALO $1 \mathrm{~m}$ & $I$ & 114 & 110 & 1.83 & 0.919 \\
\hline \multicolumn{6}{|c|}{ MACHO } \\
\hline Mt.Stromlo 50" & $R_{\mathrm{MACHO}}$ & 90 & 90 & $\cdots$ & 1.04 \\
\hline$\cdots$ & $V_{\mathrm{MACHO}}$ & 77 & 76 & $\cdots$ & $0.709 \% 0.730^{b}$ \\
\hline
\end{tabular}

${ }^{a}$ with respect to PLANET/DoPHOT model

${ }^{\mathrm{b}}$ with respect to PLANET/ISIS model 
Table 2. PLANET close-binary model of MACHO 99-BLG-47

\begin{tabular}{ccc}
\hline \hline parameters & PLANET (ISIS) + MACHO & PLANET (DoPHOT) + MACHO \\
\hline$d$ & $0.134 \pm 0.009$ & $0.121 \pm 0.009$ \\
$q$ & $0.340 \pm 0.041$ & $0.374 \pm 0.058$ \\
$t_{\mathrm{E}}$ & $163 \pm 26$ days & $183 \pm 32$ days \\
$t_{0}{ }^{a}, \mathrm{~b}$ & $1393.9331 \pm 0.0071$ & $1393.9309 \pm 0.0075$ \\
$u_{0}{ }^{\mathrm{a}}$ & $(8.6 \pm 1.1) \times 10^{-3}$ & $(7.5 \pm 1.3) \times 10^{-3}$ \\
$\alpha^{\mathrm{c}}$ & $294.99 \pm 0.25$ & $294.45 \pm 0.25$ \\
$\chi^{2}$ & 412.13 & 420.45 \\
dof & 412 & 423 \\
\hline
\end{tabular}

Note. - We note that the fact that $\chi^{2} \sim$ dof results from our rescaling of the photometric errorbars. However, we use the same scaling factor here and for models described in Table 3 so the indistinguishability between two models is not affected by this rescaling. The uncertainties for parameters are " $1-\sigma$ error bars" determined by a quadratic fit of $\chi^{2}$ surface.

${ }^{a}$ the closest approach to the binary center of mass

${ }^{\mathrm{b}}$ the Heliocentric Julian Date -2450000.

${ }^{c}$ the binary center of mass lying on the right hand side of the moving source

Table 3. PLANET wide-binary model of MACHO 99-BLG-47

\begin{tabular}{ccc}
\hline \hline parameters & PLANET (ISIS) + MACHO & PLANET (DoPHOT) + MACHO \\
\hline$d$ & $11.31 \pm 0.96$ & $12.79 \pm 1.05$ \\
$q$ & $0.751 \pm 0.193$ & $0.917 \pm 0.288$ \\
$t_{\mathrm{E}}{ }^{\mathrm{a}}$ & $220 \pm 37$ days & $253 \pm 47$ days \\
$t_{0} \mathrm{~b}, \mathrm{c}$ & $1393.9113 \pm 0.0072$ & $1393.9133 \pm 0.0074$ \\
$u_{0}{ }^{\mathrm{a}, \mathrm{b}}$ & $(6.5 \pm 1.0) \times 10^{-3}$ & $(5.50 \pm 0.92) \times 10^{-3}$ \\
$\alpha^{\mathrm{d}}$ & $295^{\circ} .46 \pm 0.24$ & $294^{\circ} .83 \pm 0.24$ \\
$\chi^{2}$ & 412.74 & 420.50 \\
dof & 412 & 423 \\
\hline
\end{tabular}

${ }^{a}$ with respect to the Einstein radius of the combined mass

${ }^{\mathrm{b}}$ The closest approach to the caustic center. See $\S 3$ for the definition of the caustic center.

${ }^{c}$ the Heliocentric Julian Date -2450000.

${ }^{d}$ the caustic center lying on the right hand side of the moving source 


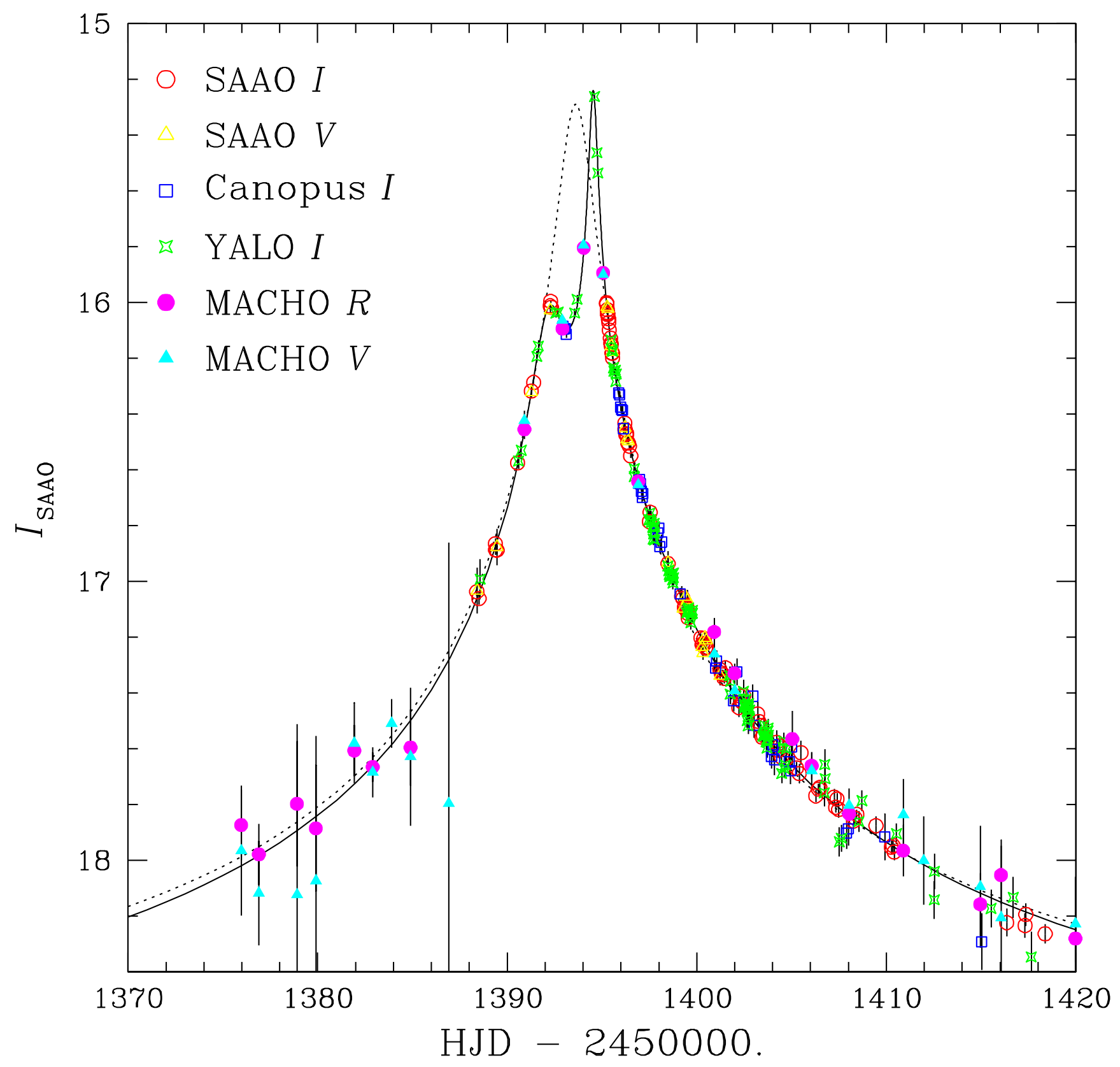

Fig. 1.- Observations and models of MACHO 99-BLG-47, all scaled to calibrated Cousins $I$-band with blending as registered by SAAO DoPHOT photometry. Shown are PLANET $I$-band from SAAO, Canopus, YALO, PLANET $V$-band from SAAO, and MACHO $V_{\mathrm{MACHO}}$ and $R_{\mathrm{MACHO}}$. The ISIS reduced PLANET points are plotted after applying the transformation to the absolute scale derived from the linear regression between difference photometry and PSF-based photometry. The solid curve shows the final close-binary lens model fit (Table 2) to the data while the dotted curve shows the "best" degenerate form of point-source/point-lens light curve (eq. [1]) fit to a high-magnification subset of the data that excludes the anomalous points near the peak. The half-magnitude offset between this curve and the data is the main observational input into the algorithm to search for the final model (see $\S 3$ ). Note that on the scale of the figure, the wide-binary solution is completely indistinguishable from the close-binary solution, i.e. the solid curve can represent both the close-binary and the wide-binary lens models equally well. 


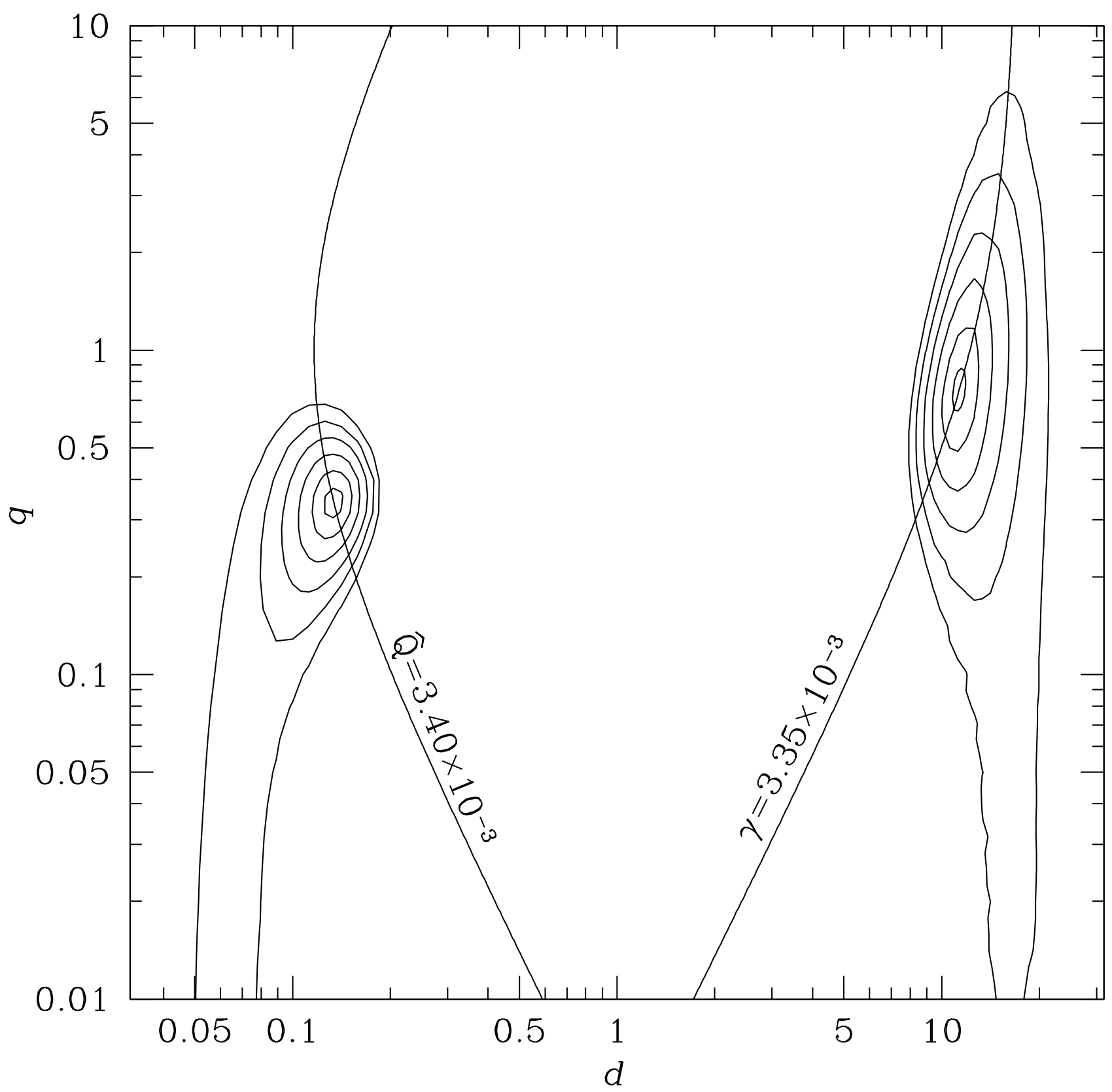

Fig. 2.- Contour plot of $\chi^{2}$-surface over $(d, q)$ space based on solutions for PLANET (ISIS) and MACHO data. The binary separation $d$ is in units of the Einstein radius of the combined mass, and the mass ratio $q$ is the ratio of the farther component to the closer component to the source trajectory (i.e., $q>1$ means that the source passes by the secondary). Contours shown are of $\Delta \chi^{2}=1,4,9,16,25,36$ (with respect to the global minimum). We find two well-isolated minima of $\chi^{2}$, one in the close-binary region, $(d, q)=(0.134,0.340)$, and the other in the wide binary-region, $(d, q)=(11.31,0.751)$ with $\Delta \chi^{2}=0.6$ and the close binary being the lower $\chi^{2}$ solution. Also drawn are the curves of models with the same $\hat{Q}$ as the best-fit close-binary model and the same $\gamma$ as the best-fit wide-binary model (see Appendix A). 


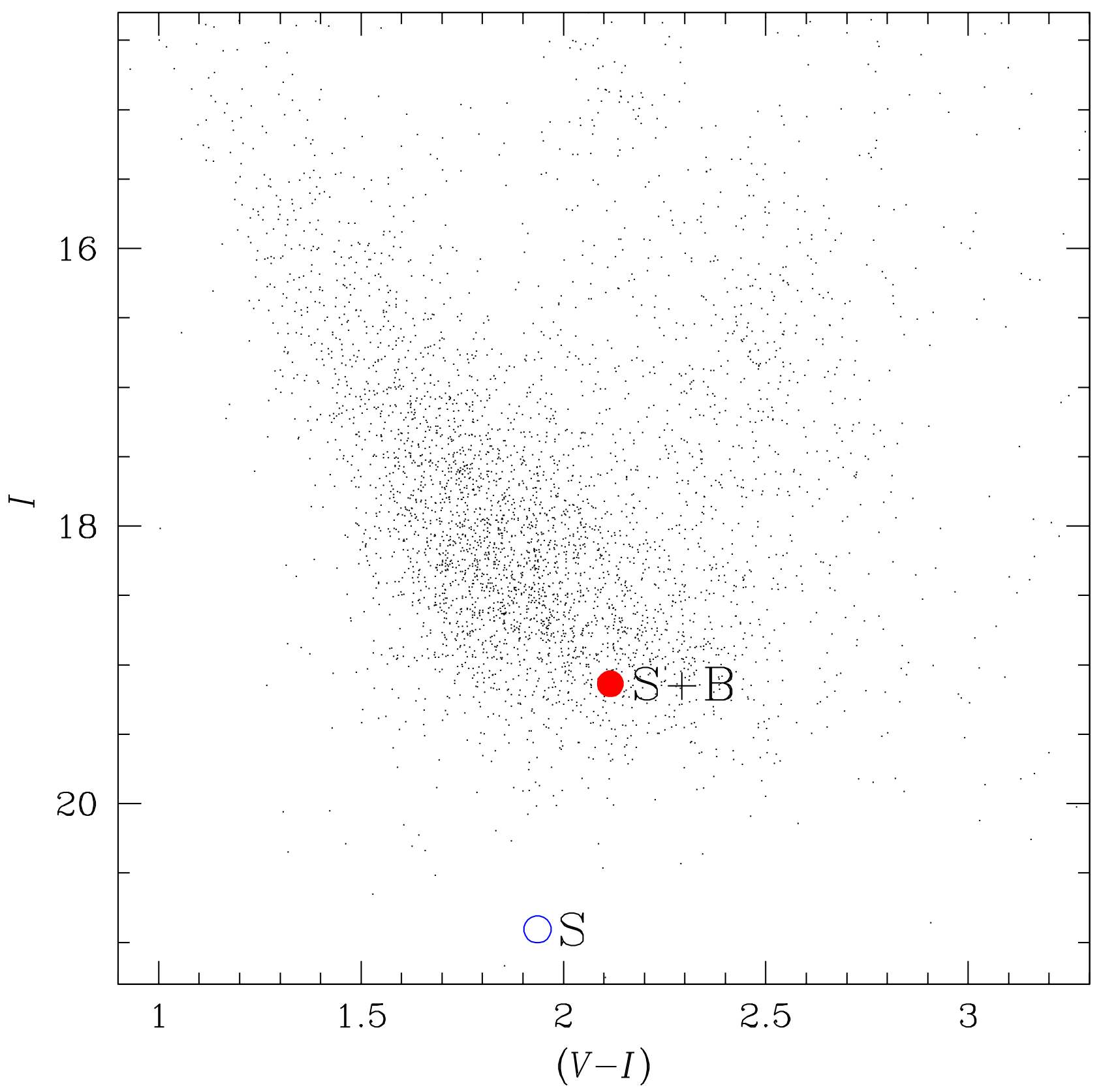

Fig. 3.- CMD of $3^{\prime} \times 3^{\prime}$ field surrounding MACHO 99-BLG-47 $\left(l=17^{\circ} 99, b=-1.96\right)$ derived from SAAO observations. Shown are the positions of the baseline "star" $(\mathrm{S}+\mathrm{B})$ and the lensed source $(\mathrm{S})$ in the absence of lensing (close-binary model). The position of the lensed source in the wide-binary model differs from this by substantially less than the size of the symbol. The errors are also smaller than the symbols. The majority of stars in the CMD are most likely turn-off stars seen at increasingly greater distances in the Galactic plane, and hence at correspondingly greater reddenings. 
Fig. 4.- Grayscale plot of the difference of the normalized flux fields, $2\left|F_{w}-F_{c}\right| /\left(F_{\mathrm{s}, w} A_{w}+F_{\mathrm{s}, c} A_{c}\right)$ of the two PLANET models around the caustic. The two fields are scaled and aligned so that the source trajectories coincide with each other. The resulting shape and the relative size of the caustics are remarkably close to each other [c.f., fig. 8 of Afonso et al. (2000) and fig. 6 of Albrow et al. (2001a)]. Also drawn are the contours of zero difference (dotted line) and 5\% difference (solid line). While the actual source trajectory $(y=0)$ naturally traces well the zero-difference line, the flux difference would also be extremely small for other trajectories through the region, except very near the caustic. 
This figure "fig4.jpg" is available in "jpg" format from: http://arxiv.org/ps/astro-ph/0201256v2 